\title{
Identification of Two Reactive Cysteine Residues in the Tumor Suppressor Protein p53 Using Top-Down FTICR Mass Spectrometry
}

\author{
Jenna Scotcher, ${ }^{1}$ David J. Clarke, ${ }^{1}$ Stefan K. Weidt, ${ }^{1}$ C. Logan Mackay, ${ }^{1}$ Ted R. Hupp, ${ }^{2}$ \\ Peter J. Sadler, ${ }^{3}$ Pat R. R. Langridge-Smith ${ }^{1}$ \\ ${ }^{1}$ SIRCAMS, School of Chemistry, University of Edinburgh, West Mains Road, Edinburgh EH9 3JJ, UK \\ ${ }^{2}$ Cell Signaling Unit, Edinburgh Cancer Research Centre, University of Edinburgh, Edinburgh, UK \\ ${ }^{3}$ Department of Chemistry, University of Warwick, Coventry, UK
}

\begin{abstract}
The tumor suppressor p53 is a redox-regulated transcription factor involved in cell cycle arrest, apoptosis and senescence in response to multiple forms of stress, as well as many other cellular processes such as DNA repair, glycolysis, autophagy, oxidative stress and differentiation. The discovery of cysteine-targeting compounds that cause re-activation of mutant p53 and the death of tumor cells in vivo has emphasized the functional importance of p53 thiols. Using a combination of top-down and middle-down FTICR mass spectrometry, we show that of the 10 Cys residues in the core domain of wild-type p53, Cys182 and Cys277 exhibit a remarkable preference for modification by the alkylating reagent $\mathrm{N}$-ethylmaleimide. The assignment of Cys 182 and Cys 277 as the two reactive Cys residues was confirmed by site-directed mutagenesis. Further alkylation of p53 beyond Cys182 and Cys277 was found to trigger cooperative modification of the remaining seven Cys residues and protein unfolding. This study highlights the power of top-down FTICR mass spectrometry for analysis of the cysteine reactivity and redox chemistry in multiple cysteine-containing proteins.
\end{abstract}

Key words: p53, Tumor-suppressor, FTICR mass spectrometry, Top-down fragmentation, Middle-down fragmentation, Cysteine reactivity

\section{Introduction}

$\mathrm{T}$ he tumor-suppressing protein p53 is a transcription factor best known for its key role in mediating cell cycle arrest, apoptosis, or senescence in response to numerous different forms of cellular stress. Over recent years, p53 has also been implicated in the regulation of many other cellular

Electronic supplementary material The online version of this article (doi:10.1007/s13361-011-0088-x) contains supplementary material, which is available to authorized users.

Correspondence to: Pat R. R. Langridge-Smith; e-mail: prrls@ed.ac.uk processes including DNA repair, glycolysis, autophagy, oxidative stress and differentiation [1-3]. It has also been shown to exert a pro-apoptotic effect independently of its transcriptional activity [4]. Despite over 30 years of extensive p53 research, comparatively little is known about the specific molecular mechanisms that allow p53 to discriminate between its different functions, and there is particular current interest in determining how p53 differentially activates expression of its target genes [5].

The DNA-binding domain of human p53 contains 10 cysteine residues (Cys124, 135, 141, 176, 182, 229, 238, 242, 275, and 277), nine of which are highly conserved (all but Cys229) [6] and three of which are involved in the coordination of a zinc ion (Cys176, 238, and 242, along with 
His179) [7]. There is clear and mounting evidence suggesting that redox-regulation of specific Cys residues in p53 plays a crucial role in controlling its activity. The redox-state of p53 cysteine residues influence its capacity to bind to consensus DNA in vitro and in vivo [8-13] and oxidation of endogenous p53 cysteines has been observed in both stressed and unstressed cells [14-16]. Proteins responsible for modulating Cys redox-state, such as thioredoxin (Trx) and redox factor 1 (ref-1), participate in the regulation of p53 activity [17, 18], and multiple signaling pathways exist that link reactive oxygen species (ROS) and reactive nitrogen species (RNS) with p53 [19, 20]. In addition, the recent discovery of cysteine-targeting electrophiles that reactivate mutant p53 in vivo, resulting in apoptosis of tumor cells, emphasizes the functional importance of p53 cysteines [21, 22].

Despite the growing interest in redox-regulated proteins, analysis of multiple Cys-containing proteins remains a challenge. Previous methods used to define the oxidation/ modification state of wild-type (wt) p53 Cys residues have not allowed site-specific information on all 10 Cys residues. Here, we show that Fourier transform ion cyclotron resonance mass spectrometry (FTICR MS) [23], combined with top-down fragmentation using collision-induced dissociation (CID) [24] and electron capture dissociation (ECD) $[25,26]$, enables simultaneous and unambiguous definition of the modification state of each Cys residue in the p53 DNA-binding domain. In top-down protein mass spectrometry, the mass-to-charge ratio $(\mathrm{m} / \mathrm{z})$ of an intact protein is first measured, followed by isolation and fragmentation of the intact molecule. Analysis of the resulting fragments permits the unambiguous assignment of combinatorial protein modifications [27, 28]. FTICR is the method of choice for top-down MS, due to its superior mass resolving power and mass accuracy, which permits the accurate mass measurement of an intact protein, and is also required to resolve and identify the many multiply-charged product ions that are generated from fragmentation of an intact protein $[29,30]$.

We have studied the reactivity of p53 cysteine residues towards alkylation with the thiol-derivatizing reagent $\mathrm{N}$-ethylmaleimide (NEM), a reagent which has previously been shown to have a concentration-dependent effect on specific DNA-binding of p53 [31]. Low concentrations of NEM resulted in increased DNA-binding, whereas higher concentrations resulted in decreased DNA-binding. The same trend is observed for alkylation of mutant p53 by the anti-tumor maleimide compound MIRA- 1 and its analogues [32]. FTICR mass spectrometry of NEM-modified p53 core domain has revealed that alkylation of the protein proceeds in distinct steps and that two cysteine residues on the p53 surface display enhanced reactivity compared with the remaining Cys residues. We hope that the results from this study will allow more targeted and focused investigations into the unknown redox-mechanisms that are involved in regulating $\mathrm{p} 53$ function.

\section{Experimental}

\section{Materials}

Unless stated otherwise, all reagents were purchased from Sigma-Aldrich. All solvents were purchased from Fisher Scientific and were of LC-MS grade purity.

\section{Recombinant Expression and Purification of p53 Core Domain}

Wild-type human p53 core domain (a.a. residues 94-312) was expressed from the vector pRSET(A) and purified as previously described [33, 34]. Briefly, E. coli C41 (DE3) cells, harboring the pRSET(A) plasmid were grown to an $\mathrm{OD}_{600} \sim 0.6-0.8$ at $37^{\circ} \mathrm{C}$ before the temperature was reduced to $22^{\circ} \mathrm{C}$, and overnight expression was induced with $1 \mathrm{mM}$ isopropyl $\beta$-D-thiogalactoside. Cells were lysed using BugBuster Protein Extraction Reagent (Novagen) and protein was purified using a HiTrap SP XL column (GE Healthcare) on an ÄKTA FPLC system. p53 concentration was determined from $\mathrm{A}_{280 \mathrm{~nm}}$ using the extinction coefficient $\varepsilon=17,130 \mathrm{M}^{-1} \mathrm{~cm}^{-1}$ [35]. Purified protein was stored at $-80^{\circ} \mathrm{C}$ in the presence of $10 \%$ glycerol.

\section{Site-Directed Mutagenesis}

Mutagenesis was performed using the QuikChange SiteDirected Mutagenesis Kit (Stratagene) according to the manufacturer's instructions. p53 Cys mutants were expressed and purified as described above.

\section{Cysteine Alkylation}

All alkylation experiments were performed in $25 \mathrm{mM}$ sodium phosphate ( $\mathrm{pH}$ 7.2), $100 \mathrm{mM} \mathrm{KCl}, 0.2 \mathrm{mM}$ EDTA, and 20\% glycerol. Buffer exchange was performed using PD MiniTrap G-25 columns (GE Healthcare). Wild-type or mutant p53 core domain $(35 \mu \mathrm{M})$ was incubated with 0 , $0.05,0.1,1,2$, or $5 \mathrm{mM} N$-ethylmaleimide (NEM) for 15 min at $22^{\circ} \mathrm{C}$ or $37^{\circ} \mathrm{C}$. At $22^{\circ} \mathrm{C}$, cysteine alkylation was quenched by the addition of dithiothreitol (DTT) to a final concentration of $25 \mathrm{mM}$. At $37^{\circ} \mathrm{C}$, alkylation was quenched by trichloroacetic acid (TCA) precipitation and protein was re-suspended in $6 \mathrm{M}$ guanidine hydrochloride, $25 \mathrm{mM}$ sodium phosphate ( $\mathrm{pH} 7.2$ ), and $25 \mathrm{mM}$ DTT. For time course experiments, $1 \mathrm{mM}$ or $0.5 \mathrm{mM}$ NEM was incubated with wild-type or mutant p53 core domain $(35 \mu \mathrm{M})$ at $22^{\circ} \mathrm{C}$ or $37^{\circ} \mathrm{C}$, respectively. Aliquots were removed at various time points and quenched with DTT or TCA precipitation. Quenched samples were analyzed by on-line liquid chromatography FTICR mass spectrometry (LC-FTICR MS).

\section{Proteolytic Digestion and Sample Preparation}

For middle-down or bottom-up MS, quenched samples of wild-type p53 treated with $0.1 \mathrm{mM}$ NEM were incubated 
with Lys-C (Roche) or trypsin (Promega) for $4 \mathrm{~h}$ at $37^{\circ} \mathrm{C}$ at an enzyme to protein ratio of approximately $1: 20$ by weight. Following Lys-C digestion, precipitated protein was collected by centrifugation at $13,000 \mathrm{rpm}$ and resuspended in $6 \mathrm{M}$ guanidine hydrochloride, $25 \mathrm{mM}$ sodium phosphate (pH 7.2), and $10 \mathrm{mM}$ DTT. Prior to mass spectrometry, all digested samples were desalted and buffer-exchanged into $50 \%$ acetonitrile and $0.6 \%$ formic acid using $\mathrm{C}_{18}$ ZipTip pipette tips (Millipore).

\section{On-line LC-FTICR MS}

On-line liquid chromatography was used as a desalting step prior to mass spectrometry. An Ultimate 3000 HPLC system (Dionex) was equipped with a monolithic PS-DVB $(500 \mu \mathrm{m} \times 50 \mathrm{~mm})$ reverse-phase analytical column (Dionex) and coupled to a Triversa NanoMate (Advion BioSciences) for nano-electrospray ionization (nESI). Protein $(5 \mathrm{pmol})$ was loaded onto the column (maintained at $60^{\circ} \mathrm{C}$ ) followed by a 10 min linear gradient from $2 \%$ to $70 \%$ acetonitrile (flow rate $20 \mu \mathrm{L} / \mathrm{min}$ ). Prior to top-down MS of 2NEM-p53, LC eluent containing intact NEM-modified p53 core domain was manually collected and directly infused into the mass spectrometer.

\section{FTICR Mass Spectrometry}

All mass spectra were recorded on an apex-Qe or solariX FTICR mass spectrometer equipped with a $12 \mathrm{~T}$ superconducting magnet (Bruker Daltonics). Direct infusion of protein/peptide samples (typical concentration $\sim 10 \mu \mathrm{M}$ ) was achieved using the Triversa NanoMate for denatured spectra, or a solariX ESI source (Bruker Daltonics) for native protein spectra. Native spectra were recorded from $50 \mathrm{mM}$ ammonium acetate (pH 7) and 10\% methanol. Transient data size was 512 Kword for each acquisition. Ions were detected over $550-3800 \mathrm{~m} / \mathrm{z}$ for denatured conditions (transient length $0.8 \mathrm{~s}$ ) and $800-4000 \mathrm{~m} / \mathrm{z}$ for native conditions (transient length $1.4 \mathrm{~s}$ ).

\section{Tandem FTICR Mass Spectrometry}

MS/MS was performed on an apex-Qe FTICR mass spectrometer equipped with a $12 \mathrm{~T}$ superconducting magnet. Specific ion species were isolated using the mass resolving quadrupole and MS/MS was performed using collisioninduced dissociation (CID) or electron capture dissociation (ECD). Typical collision energy values for top-down and middle-down MS were between -10 and $-22 \mathrm{eV}$. The collision energy used to fragment the tryptic peptide VCACPGR was $-29 \mathrm{eV}$. For ECD fragmentation, $1.8 \mathrm{~A}$ was applied to the hollow dispenser cathode (HeatWave Technologies) and pulse lengths were between 8 and $18 \mathrm{~ms}$; $100-500$ transients were averaged for fragmentation spectra.

\section{Data Analysis}

All spectra were externally calibrated using ES tuning mix (Agilent Technologies) and analyzed using DataAnalysis software (Bruker Daltonics). Neutral spectra were created using Maximum Entropy deconvolution (Bruker Daltonics). Isotope distributions of specific charge states were predicted from theoretical empirical formulas. These were overlaid upon the recorded experimental data as scatter plots, with the theoretical apex of each isotope peak designated by a circle. Relative ratios of alkylated forms of p53 were calculated from peak heights [36]. The SNAP 2.0 algorithm was used for automated peak picking of top-down and middle-down fragmentation data and the resulting mass lists were searched against the relevant primary sequences using ProSight PTM [37] or Biotools (Bruker Daltonics) software. Error tolerances were $10 \mathrm{ppm}$.

\section{Results and Discussion}

\section{Concentration and Time-Dependence of p53 Alkylation}

Accurate mass measurement of $\mathrm{p} 53$ core domain prior to alkylation confirmed that each of the 10 cysteine residues was in the reduced form (Cys-SH; Fig. 1). In addition, analysis of the protein under native MS conditions (i.e., neutral $\mathrm{pH}$ and $\sim 10 \%$ organic solvent to aid the desolvation process) revealed the presence of one $\mathrm{Zn}^{2+}$ ion per protein molecule (Fig. 1). This observation is consistent with the known metal binding properties of $\mathrm{p} 53$ and indicates correct folding of the p53 DNA-binding domain [38, 39]. p53 core domain $(35 \mu \mathrm{M})$ was incubated with increasing concentrations of NEM for $15 \mathrm{~min}$ at $22^{\circ} \mathrm{C}$. The reaction was quenched by the addition of dithiothreitol (DTT) and the degree of thiol alkylation was monitored by FTICR MS. The resulting deconvoluted neutral mass spectra (Fig. 2a) clearly show that p53 is predominantly modified with two NEM groups (2NEM-p53) at low concentrations of alkylating reagent $(\mathrm{NEM}<1 \mathrm{mM})$. At higher concentrations (NEM $>1 \mathrm{mM}$ ), p53 was modified with ten NEM groups, i.e., all Cys residues were alkylated. Complete conversion to 10NEM-p53 was observed with $5 \mathrm{mM}$ NEM (see Supplementary Figure S1 for accurate mass measurement of modified forms of $\mathrm{p} 53$ ). It was notable that only relatively small amounts of species with intermediate stages of alkylation were detected.

To monitor the time-dependence of alkylation, p53 was incubated with $1 \mathrm{mM} \mathrm{NEM}$ and the reaction was quenched with DTT at various time points followed by FTICR MS. Rapid alkylation of two cysteine residues was apparent; after $5 \mathrm{~s}$ the neutral mass spectra (Fig. $2 \mathrm{~b}$ ) showed that ca. $32 \%$ of p53 was monoalkylated and $63 \%$ dialkylated. The proportion of 2NEM-p53 increased to ca. $85 \%$ after $15 \mathrm{~s}$ and ca. $100 \%$ by 1 min. Alkylation of a third Cys residue (i.e., formation of 3NEM-p53) was not apparent until the 5-min 

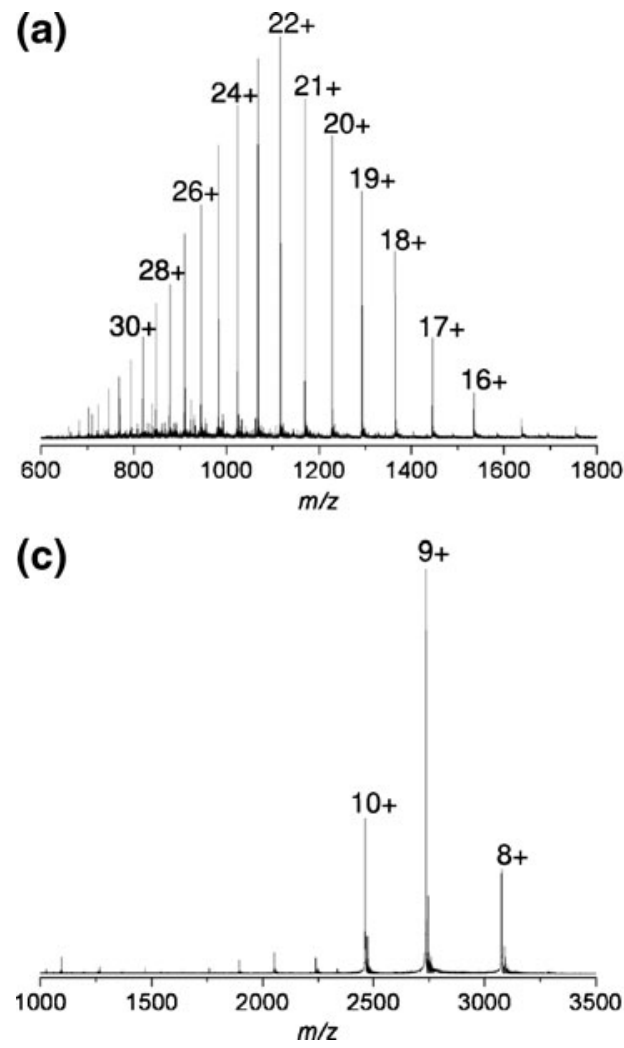

(b)

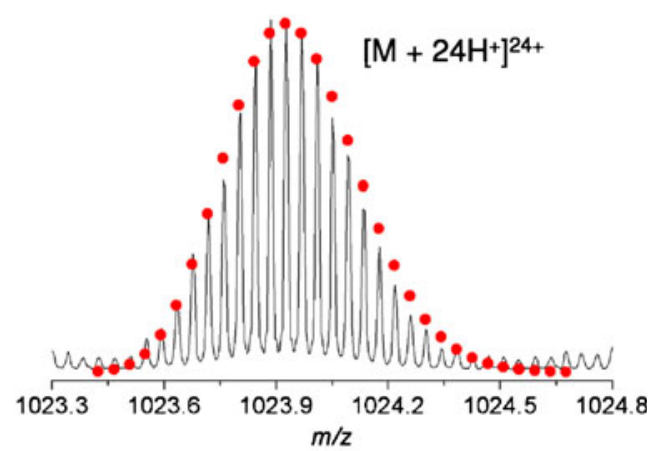

(d)

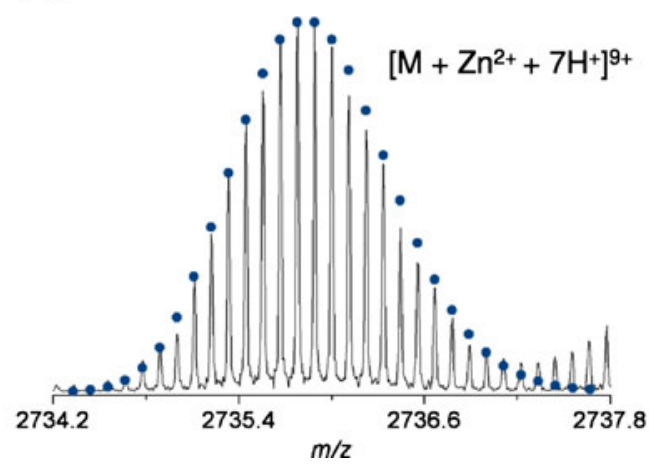

Figure 1. Accurate mass measurement of p53 core domain by FTICR MS. (a) nESI mass spectrum of p53 recorded under denaturing conditions. (b) $24+$ charge state of denatured p53. The experimental isotope distribution is consistent with the theoretical isotope distribution of apo-p53 core domain with each cysteine residue in the reduced form (empirical formula $\left[\mathrm{C}_{1055} \mathrm{H}_{1697} \mathrm{~N}_{321} \mathrm{O}_{324} \mathrm{~S}_{16}\right]^{24+} ; \bullet ; 5$ ppm mass measurement error (MME); 80,000 resolving power (RP) at $\mathrm{m} / \mathrm{z}$ 1023.9). (c) ESI mass spectrum of p53 core domain recorded under native conditions. (d) 9+ charge state of native p53. The experimental isotope distribution is consistent with the theoretical isotope distribution of p53 core domain bound to one $\mathrm{Zn}^{2+}$ ion (empirical formula $\left[\mathrm{C}_{1055} \mathrm{H}_{1680} \mathrm{~N}_{321} \mathrm{O}_{324} \mathrm{~S}_{16} \mathrm{Zn}\right]^{9+} ; \bullet ; 1$ ppm MME; 135,000 RP at $\mathrm{m} / \mathrm{z} 2735.9$ )

time point, after which direct conversion of 3NEM-p53 to 10NEM-p53 was observed.

\section{p53 Alkylation at Physiological Temperature}

The p53 core domain is known to have a low thermodynamic stability [40] and to unfold at body temperature [41]. Therefore, concentration and time dependence of p53 alkylation was investigated at $37^{\circ} \mathrm{C}$ (Supplementary Figure S2). A similar pattern of p53 alkylation was observed, namely, initial alkylation of two Cys residues followed by direct conversion of 3NEM-p53 to 10NEM-p53 upon increasing NEM concentration or time. At $37^{\circ} \mathrm{C}$, complete conversion to 10NEM-p53 was observed with $0.5 \mathrm{mM}$ NEM, and ca. $80 \%$ of p53 had been fully alkylated by the 5 -min time point.

\section{Identification of Reactive Cysteine Residues}

In order to identify the two preferentially alkylated Cys residues using top-down FTICR mass spectrometry, individual charge states $(26+, 29+, 31+$ and $32+)$ of the 2NEM-p53 species were sequentially isolated and frag- mented using CID or ECD (Fig. 3). The resulting fragment masses were searched against theoretical fragment mass lists for each permutation of Cys-NEM (assigned product ions are listed in Supplementary Tables S1 and S2). Taken together, top-down fragmentation data resulted in $51 \%$ sequence coverage, and allowed assignment of the oxidation state of eight out of the 10 cysteine residues within p53 (Fig. 3c). No fragment ions were observed between Cys275 and Cys277 and, thus, the state of these two residues could not be distinguished unambiguously. Top-down FTICR MS of 2NEM-p53 indicated that Cys182 was alkylated and the remaining NEM group was located on either Cys275 or Cys277.

In order to increase sequence coverage over the internal region of the p53 core domain and to obtain a diagnostic fragment between Cys275 and Cys277, 2NEM-p53 was cleaved with Lys-C protease. Such enzymatic or chemical cleavage of proteins into large peptides prior to tandem mass spectrometry provides a useful strategy (termed middledown mass spectrometry) for increasing sequence coverage compared with fragmentation of the intact protein [42]. LysC cleavage resulted in a 127 amino-acid peptide, from Gln165 to Lys291, containing the seven cysteine residues 

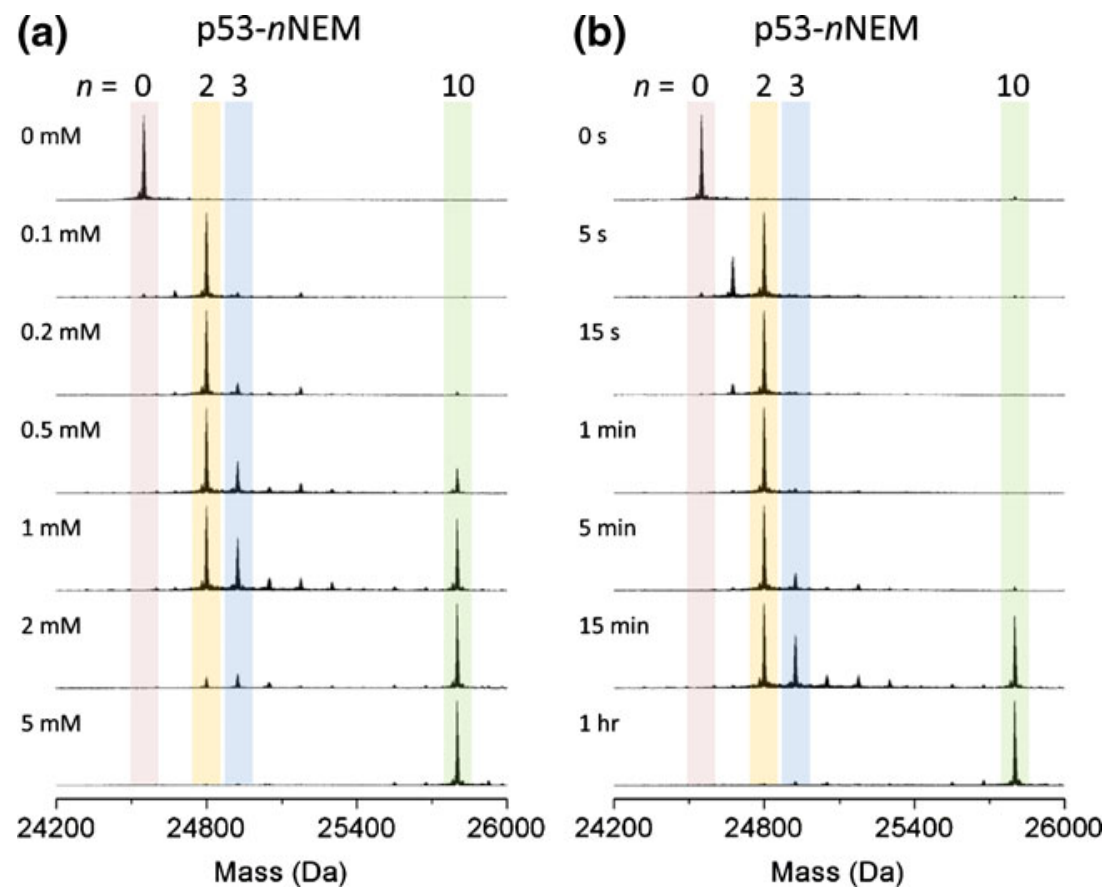

Figure 2. Reaction of p53 core domain with $N$-ethylmaleimide. (a) NEM titration. p53 was incubated with varying concentrations of NEM for 15 min. Deconvoluted neutral mass spectra are shown. (b) Cysteine alkylation over time. p53 was incubated with $1 \mathrm{mM} \mathrm{NEM}$ and deconvoluted neutral mass spectra are shown for various time points

from Cys 176 to Cys277. Incubation of 2NEM-p53 with Lys-C resulted in a protein precipitate. This was resuspended in $6 \mathrm{M}$ guanidine hydrochloride, desalted, and analyzed by FTICR mass spectrometry (Supplementary Figure S3).

Individual charge states $(13+, 14+, 18+, 19+, 20+$, and $21+$ ) of the 127 amino-acid peptide modified with two NEM groups $\left(2 \mathrm{NEM}-\mathrm{p} 53_{\text {Lys-C }}\right)$ were sequentially isolated and fragmented using CID or ECD (Fig. 4; assigned product ions are listed in Tables S3 and S4). Fragmentation of 2NEM-p $53_{\text {Lys-C }}$ resulted in an increase in sequence coverage from 39\% (achieved for the region Gln165 to Lys291 in intact 2NEM-p53) to $67 \%$, and allowed the oxidation state of each of the seven cysteine residues to be determined (Fig. 4c). Increased fragmentation between Cys176 and Cys 182 confirmed the previous assignment of a NEM group on Cys182. Middle-down MS also resulted in a fragment ion between Cys275 and Cys277 $\left(y_{16}{ }^{2+}\right.$; Figure S3), which supported assignment of the second NEM group on Cys277. This assignment was confirmed by bottom-up MS using a tryptic digest of 2NEM-p53. The peptide mixture was directly infused into the mass spectrometer without prior separation. Five out of the seven cysteine-containing tryptic peptides were detected (Supplementary Figure S4). Hence, use of the bottomup approach alone does not allow the oxidation state of all ten cysteine residues in p53 to be determined. However, the peptide containing Cys275 and Cys277 (VCACPGR) was detected and its mass-to-charge ratio $(\mathrm{m} / \mathrm{z})$ corresponded to the addition of one NEM group (Figure S4). Fragmentation of this peptide by CID confirmed the assignment of the NEM group on Cys277 (Figure S4 and Table S5).

\section{Alkylation of p53 Cysteine Mutants}

In order to test the FTICR MS fragmentation results showing that Cys 182 and Cys 277 are preferentially alkylated in p53, alkylation experiments were repeated with the p53 cysteineto-serine mutants $\mathrm{C} 182 \mathrm{~S}, \mathrm{C} 277 \mathrm{~S}$, and the double mutant C182/277S. Analysis of the mutants under native MS conditions confirmed that each variant was bound to one $\mathrm{Zn}^{2+}$ ion, thus indicating that the mutations did not have detrimental effects on p53 tertiary structure (Supplementary Figure S5).

The NEM titration and time course experiment were carried out for each mutant at $22^{\circ} \mathrm{C}$ (Fig. 5). As expected, for $\mathrm{C} 182 \mathrm{~S}$ and $\mathrm{C} 277 \mathrm{~S}$ only one cysteine residue was preferentially alkylated, and for the double mutant $\mathrm{C} 182$ / $277 \mathrm{~S}$, no preferential alkylation was observed. In both single mutants, direct conversion to 9NEM-p53 was observed upon alkylation of a second Cys residue, and in the double mutant, direct conversion to 8NEM-p53 was observed upon alkylation of one Cys residue. For each mutant, the formation of fully alkylated species became notable at $1 \mathrm{mM}$ NEM (at $15 \mathrm{~min}$ ), similar to wild-type p53.

\section{Preferential Alkylation of Cys 182 and Cys277}

Mass spectrometry of p53 core domain incubated with the alkylating reagent NEM under various conditions revealed that alkylation of p53 cysteine residues proceeds in distinct steps. Initially, two cysteine residues are rapidly modified to form a stable dialkylated species. An increase in NEM 
(a)

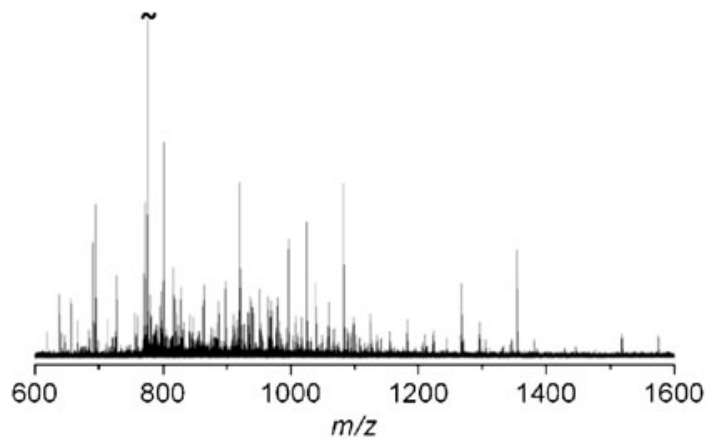

(b)

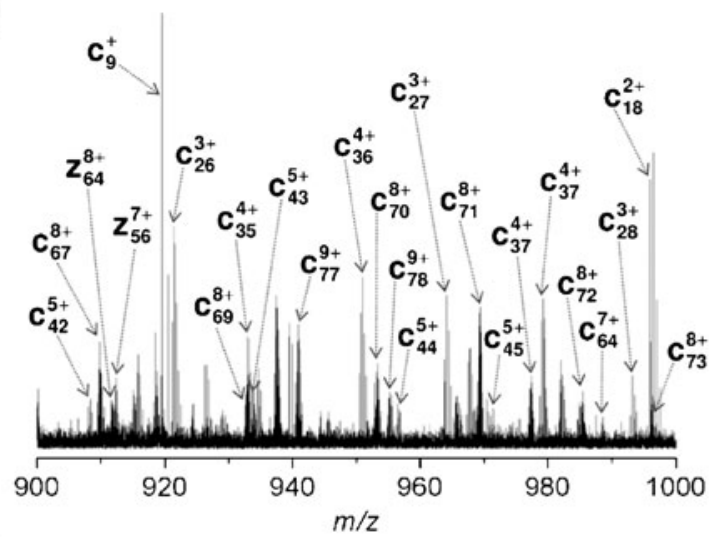

(c)

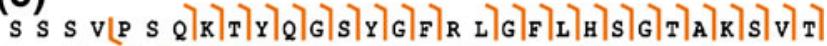
C T Y Y S STP

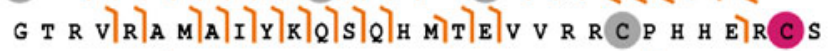
LD D G I A P P Q H L I R VEGNLRVEYLD D R T FR

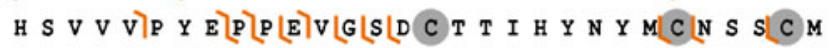
$G|G M| N R \mid R \quad P$ I L T I I I T T L $\lfloor E \backslash D\lfloor S\lfloor S\lfloor G\rfloor N\lfloor L L\lfloor G R|N\rangle S\lfloor F\lfloor E \vee R$ V C A C P G R D R R R T $\lfloor E\lfloor E\lfloor E\lfloor N\lfloor L\lfloor R\lfloor K\lfloor K\lfloor G \mid E\lfloor P$ H T KLR A L P N N T

reduced Cys NEM-Cys NEM or reduced Cys

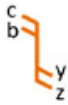

Figure 3. Top-down FTICR MS of 2NEM-p53. (a) Electron capture dissociation (ECD) mass spectrum of the $32+$ charge state $(\mathrm{m} / \mathrm{z} 776)$ of 2NEM-p53. (b) Expansion of the region $\mathrm{m} /$ $z$ 900-1000. Assigned $c$ and $z$ ions are annotated. (c) Fragment map displays ions resulting from CID and ECD fragmentation of intact p53 core domain (Ser94 -Thr312) modified with two NEM groups. Top-down FTICR MS of 2NEM-p53 shows that Cys182 is alkylated and the second NEM group is located on either Cys275 or Cys277

concentration or reaction time leads to alkylation of a third Cys residue, which initiates rapid alkylation of the remaining seven Cys residues. Alkylation of wild-type p53 was repeated at $37^{\circ} \mathrm{C}$ and the same trend was observed, albeit at a faster rate. The use of top-down and middle-down FTICR MS allowed the unambiguous identification of Cys 182 and Cys 277 as the sites of preferential alkylation. These assignments were confirmed by alkylation and MS analysis of the p53 mutants C182S, C277S, and C182/277S. (a)

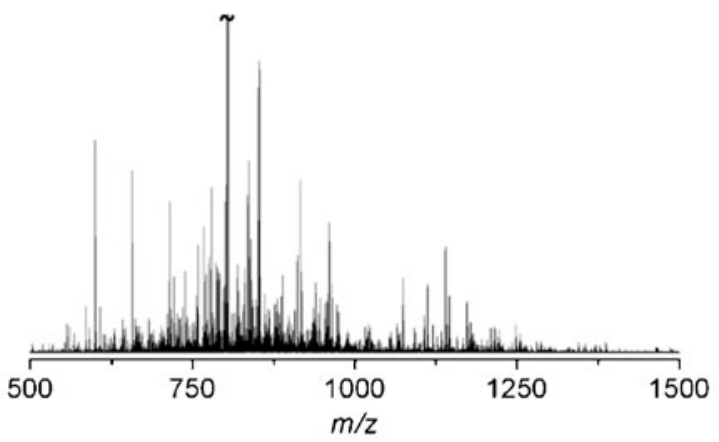

(b)

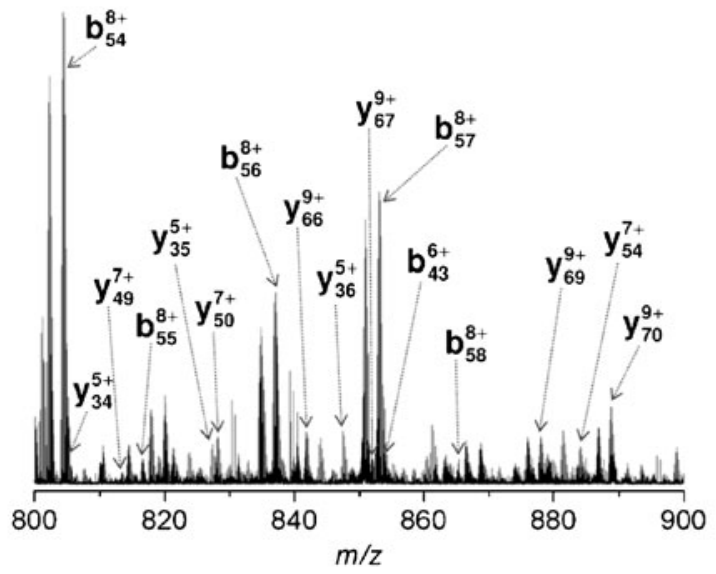

(c)

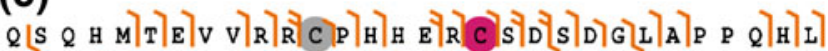
I TR V E

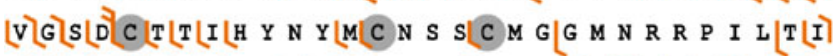

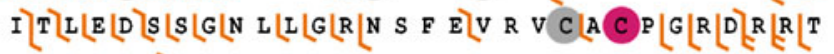
$[E\lfloor E\lfloor E\lfloor N \quad L \quad R \backslash K$

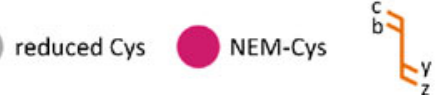

Figure 4. Middle-down FTICR MS of 2NEM-p53. (a) Collision-induced dissociation (CID) mass spectrum of the $21+$ charge state $(\mathrm{m} / \mathrm{z} 741)$ of 2 NEM-p53 $3_{\text {Lys-c. }}$ (b) Expansion of the region $\mathrm{m} / \mathrm{z} 800-900$. Assigned $b$ and $y$ ions are annotated. (c) Fragment map showing ions resulting from CID and ECD fragmentation of p53 Lys-C peptide Gln165Lys291 modified with two NEM groups. Middle-down FTICR MS shows that Cys277 is alkylated and confirms the alkylation of Cys182

Solvent accessibility values for each of the 10 thiol groups in p53 are listed in Table 1. These were determined from the Xray crystal structure of the p53 core domain (PDB ID 2OCJ) using Accelrys Discovery Studio 2.5 software [43]. Cys 182 and Cys 277 are the most accessible residues, accounting for their preferential reactivity with alkylating reagent. The sulfur atoms in Cys182 and Cys277 are significantly more accessible than the next most exposed sulfur atom, that of Cys229 (accessibility values are 39.34 and 43.34 for Cys182 and Cys277 respectively, and 14.82 for Cys229). It can clearly be seen in the surface contour representation of the crystal structure of p53, shown in Fig. 6, that Cys182 and Cys277 are the most accessible cysteine residues on the protein surface. 
(a) p53C182S- $n$ NEM

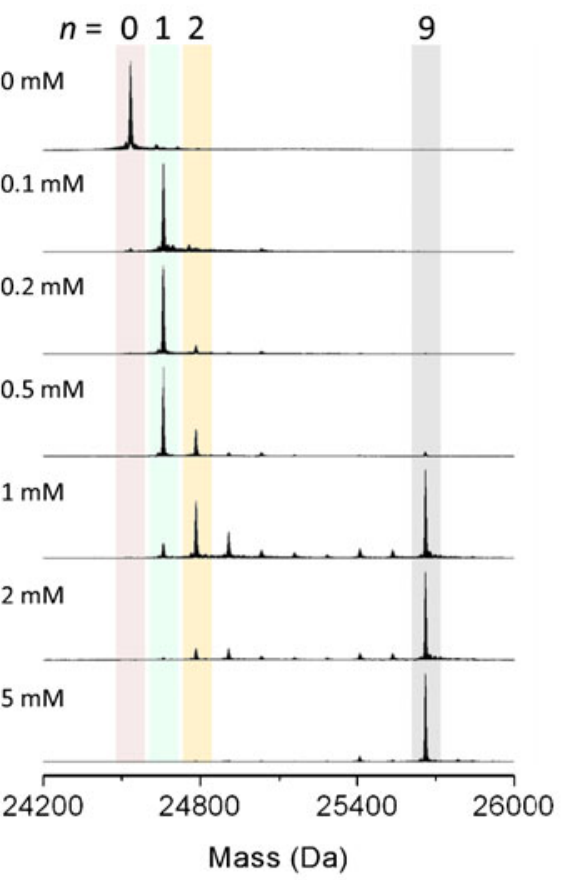

(b) $\mathrm{p} 53 \mathrm{C} 182 \mathrm{~S}-\mathrm{nNEM}$

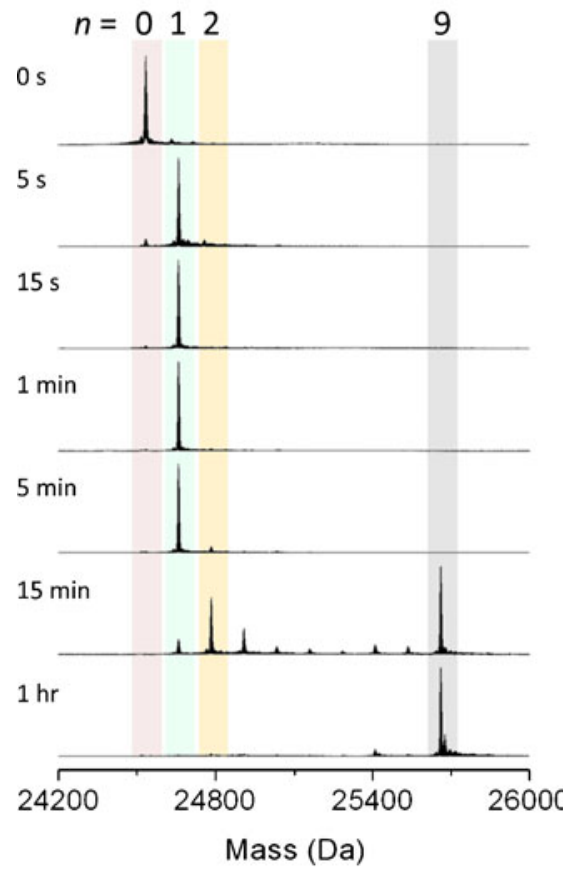

p53C277S-nNEM

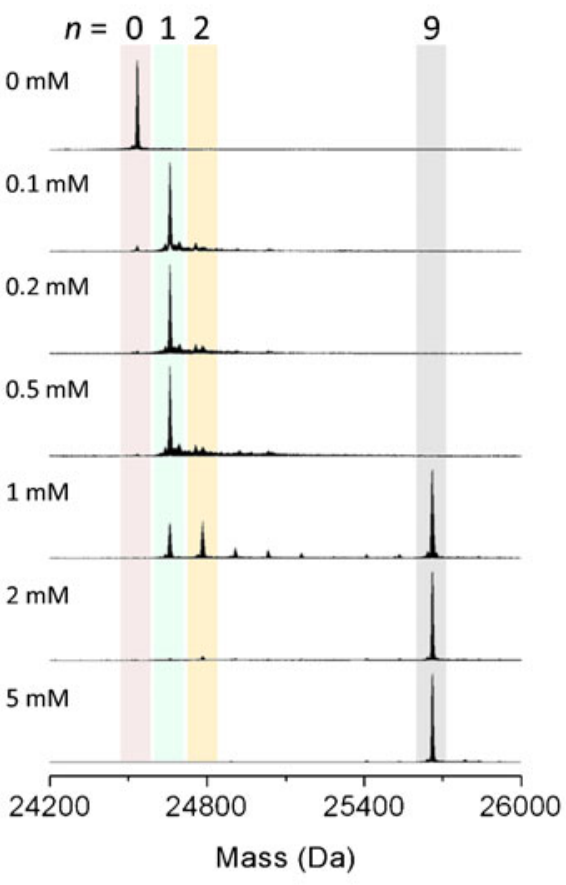

p53C277S-nNEM

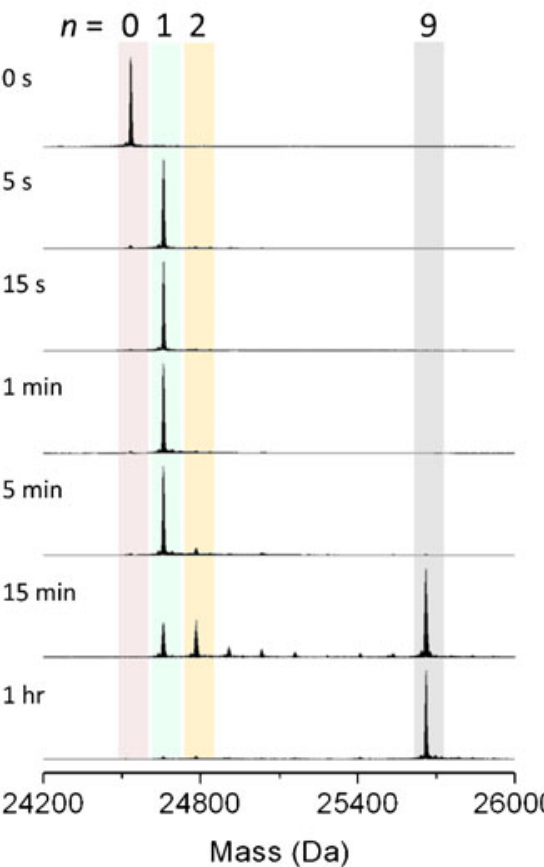

p53C182/277S-nNEM

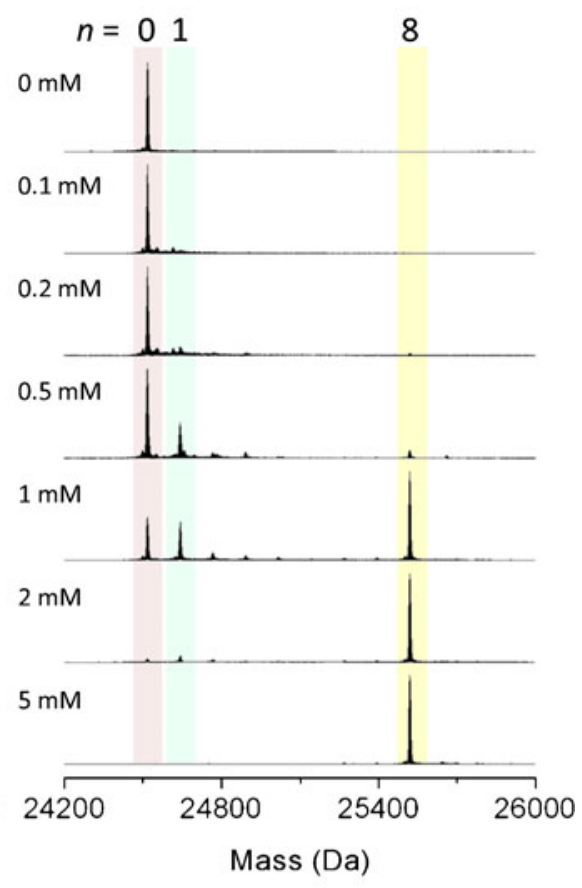

p53C182/277S-nNEM

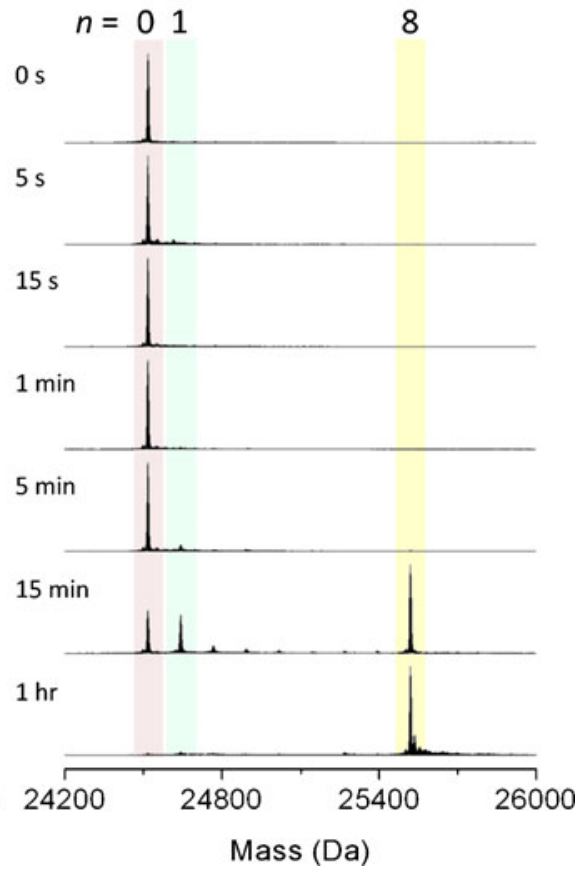

Figure 5. Reaction of $N$-ethylmaleimide with p53 core domain mutants C182S, C277S, and C182/277S. (a) NEM Titrations. (b) Alkylation over time. Reaction conditions were the same as described for wild-type p53. Deconvoluted neutral mass spectra are shown

During the preparation of this manuscript, X-ray crystallography data was reported by Kaar et al. [44], which is in agreement with the p53 FTICR mass spectrometry results presented here. Stabilised p53 core domain (T-p53C; an engineered $\mathrm{p} 53$ variant that contains the four mutations M133L/V203A/N239Y/N268D and displays increased ther- mostability compared with the wt protein [45]) containing the cancer-derived mutation Y220C was treated with an electrophilic compound, 3-benzoylacrylic acid, and the resulting crystal structure revealed pronounced electron density surrounding cysteine residues 182, 229, and 277. However, the density was not sufficient to model the 
Table 1. Solvent accessibility of thiol groups in the p53 core domain

\begin{tabular}{cc}
\hline Cysteine & Solvent accessibility $^{a}$ \\
\hline 124 & 6.27 \\
135 & 0 \\
141 & 0 \\
176 & 2.85 \\
182 & 39.34 \\
229 & 14.82 \\
238 & 1.14 \\
242 & 11.97 \\
275 & 0 \\
277 & 43.34 \\
\hline
\end{tabular}

${ }^{\mathrm{a} C}$ Calculated using Accelrys Discovery Studio 2.5 software from the X-ray crystal structure of the p53 core domain (PDB ID 2OCJ)

modifications unambiguously. Kaar et al. [44] went on to use a bottom-up MS approach to investigate the order of cysteine reactivity in T-p53C-Y220C with 3-benzoylacrylic acid. They reported that they were able to map each Cys residue using MALDI-TOF MS and LC-MS/MS. Curiously, they found Cys124 and Cys141 to be the most reactive cysteine residues. This is in conflict with both their X-ray crystallography data and the top-down FTICR MS results described here. Intriguingly, Velu et al. [46] also concluded that Cys141 is the most reactive cysteine residue in p53, after using a bottom-up MS approach to investigate cysteine alkylation. Cys141 has a solvent accessibility value of zero, and the X-ray crystal structure of the p53 core domain shows that it is buried within two sides of the $\beta$-sheet sandwich (see Table 1 and Fig. 6). A major draw-back of bottom-up MS is that when analysing peptides originating from more than one protein isoform, the 'parent' isoform from which a peptide originated is unknown. Therefore, investigating the order of Cys reactivity using a bottom-up approach requires that an individual isoform of modified protein is completely pure prior to proteolytic digestion. It may be that tryptic peptides originating from p53, which was alkylated beyond Cys182, 229 , and 277, were present in the peptide samples analysed by Kaar et al. [44] and Velu et al. [46], and resulted in the misassignment of Cys reactivity.

Interestingly, both Cys182 and Cys277 have previously been implicated in redox-regulation of p53. Cys277 is a DNA-binding residue [7] and oxidation of this residue has been implicated in differential gene recognition [11]. Cys277 has also been identified as a possible substrate for selenomethionine (SeMet)-dependent redox-regulation of p53 [15], and it has been postulated that the redox-state of Cys277 serves as a switch to activate DNA repair machinery [47]. Furthermore, an electrophilic cyclopentenone prostaglandin, 15-deoxy- $\Delta^{12,14}$-prostaglandin $\mathrm{J}_{2}\left(15 \mathrm{~d}-\mathrm{PGJ}_{2}\right)$, has been shown to bind to Cys277 in vivo, leading to an increase in p53 stability and reduction of its transcriptional activity [48]. Cys 277 is located in the conserved p53 motif BOX-V (amino acid residues 272-288). BOX-V controls the specific activity of p53 by binding to enzymes that mediate either p53 phosphorylation or ubiquitination [49]. Interestingly, redox-regulation of a cysteine residue in the $\mathrm{NF}-\kappa \mathrm{B}$ related protein, c-Rel, regulates phosphorylation of the protein, which in turn regulates its transcriptional activity [50]. It is entirely plausible that Cys277 plays a similar role in p53.

Cys182 has recently been shown to be particularly susceptible to diamide oxidation in vivo. Held et al. [16] used a differential alkylation approach, along with proteolysis and multiple reaction monitoring (MRM) peptide mass spectrometry, to identify redox sensitive cysteines in endogenous p53. Only seven of the 10 Cys residues could be monitored using this approach (all but Cys229, 238, and 242). Incomplete protein sequence coverage is a problem common to peptide-based proteomic strategies for mapping post-translational modifications, and is one of the principal reasons for the growing recognition of the power of FTICR MS for studies on intact proteins. (a)

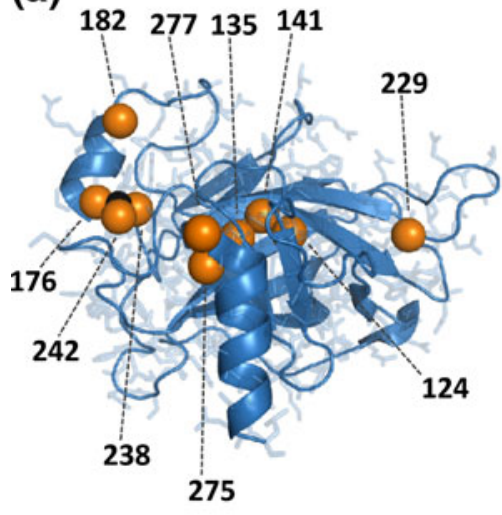

(b)

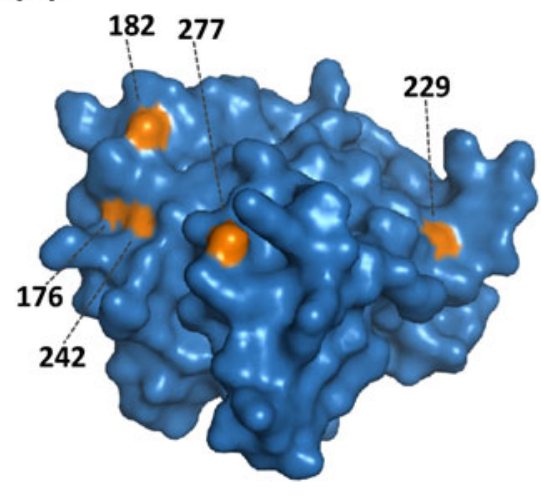

(c)

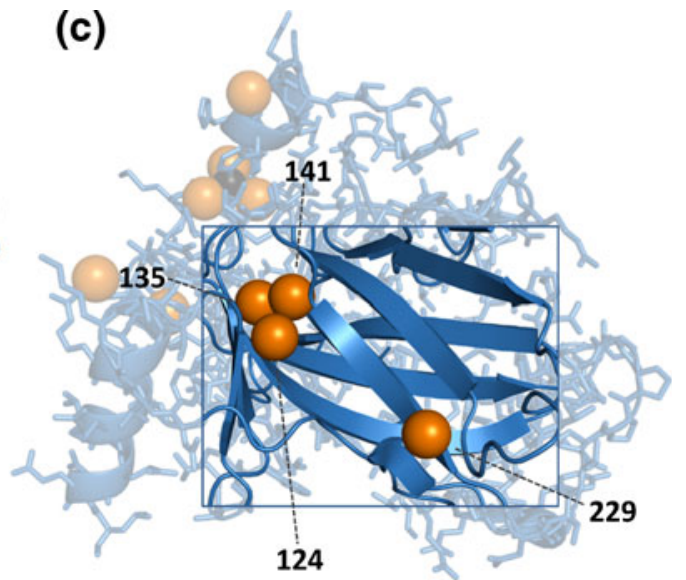

Figure 6. X-ray crystal structure of human p53 core domain (PDB ID 2OCJ; figures generated using PyMOL). (a) Ribbon diagram highlighting p53 cysteine residues. Sulfur atoms are shown (orange). Cys176, 238, and 242 are involved in the coordination of a zinc ion. (b) Surface contour of the p53 core domain. The thiol groups of Cys176, 182, 229, 242. and 277 are surface-exposed. (c) Alternative orientation of p53 core domain highlighting the anti-parallel $\beta$-sheet sandwich and position of Cys229, 124, 135, and 141 


\section{Identification of the Third Site of Alkylation}

Since alkylation beyond Cys182 and Cys277 triggers cooperative alkylation of the remaining cysteine residues, we sought to identify the third site of NEM alkylation in wildtype p53 using top-down FTICR MS. After $1 \mathrm{mM}$ NEM treatment for $15 \mathrm{~min}$, individual charge states $(23+$ and $24+)$ of 3NEM-p53 were sequentially isolated and fragmented using CID, and the resulting fragment masses were searched against theoretical fragment mass lists for p53 core domain modified with three NEM groups (Supplementary Figure S6; assigned product ions are listed in Table S6). None of the fragment ions was compatible with assignment of a NEM group on Cys124, 135, 141, or 176. However, one fragment ion was observed that supported assignment of the third NEM on Cys229, Cys238, or Cys242 $\left(b_{169}{ }^{16+}\right.$; Figure S6). Since Cys238 and 242 are zinc-binding residues it seems unlikely that either of these is the third site of alkylation. One fragment ion was also observed that supported placement of the third NEM on Cys275 $\left(y_{41}{ }^{5+}\right.$; Figure S6). Hence, Cys 229 and Cys 275 are the most likely candidates for the third site of alkylation.

\section{Disruption of Secondary Structure and Protein Unfolding}

Modification of a third Cys residue in wild-type p53 (or a second Cys in C182S and C277S, or the first Cys in C182/277S) initiated a cascade of cysteine alkylation resulting in a fully alkylated species. Top-down FTICR MS of 3NEM-p53 revealed that Cys229 and Cys275 are the most likely candidates for the third site of alkylation. Interestingly, X-ray crystallography data has previously indicated that Cys229 is modified by the electrophilic compound 3-benzoylacrylic acid [44]. Both Cys229 and Cys275 are located at the N-terminal and C-terminal ends, respectively, of strands of the anti-parallel $\beta$-sheet sandwich. We postulate that alkylation of either of these residues disrupts this stabilizing structural feature and thus allows access of NEM to the cluster of buried cysteines 124,135 , and 141, which are located in between the two sides of the $\beta$-sandwich (Fig. 6c). Interestingly, Cys124, 135, and 141 are all located at the beginning or end of $\beta$-strands. Alkylation of any one of these cysteines may result in severe disruption of p53 secondary structure and is likely to be responsible for the observed rapid alkylation of the remaining cysteine residues.

Zinc is required for the correct folding and specific DNAbinding activity of p53 [17, 38, 39]. Notably, the observed cascade of cysteine reactivity includes alkylation of all three zinc-binding cysteines, indicating that hyper-alkylation of p53 causes extensive unfolding of the core domain. This has implications for the reactivation of mutant p53 with cysteine-targeting compounds such as PRIMA-1 and MIRA-1 [21].

\section{Conclusion}

Selective modification of cysteine residues is a fundamental requirement in cellular redox-signaling [51]. Here, we have clearly and unambiguously shown that Cys182 and Cys277 in p53 are selectively alkylated by $N$-ethylmaleimide and are thus the most likely candidates for controlling p53 activity via redox-regulation. The p53 core domain has a low thermodynamic stability [40] and is known to unfold at body temperature [41]. However, we have shown that selective alkylation of $\mathrm{p} 53$ occurs at $37^{\circ} \mathrm{C}$, providing further support for the role of Cys182 and Cys277 in p53 redoxregulation. These results demonstrate the power of FTICR mass spectrometry for the study of multiple Cys-containing proteins.

\section{Acknowledgments}

The authors thank Penka Nikolova (King's College London) for donation of the p53 core domain expression vector and Dominic Campopiano (University of Edinburgh) for use of FPLC equipment. The authors acknowledge support of this research by the RCUK Interdisciplinary Research Collaboration: RASOR. They thank Professor Sir Kenneth Murray and Drs. Dominic Campopiano and Andrew Alexander for their critical comments on this manuscript.

\section{References}

1. Levine, A.J., Oren, M.: The first 30 years of p53: growing ever more complex. Nat. Rev. Cancer 9, 749-758 (2009)

2. Vousden, K.H., Lane, D.P.: p53 in health and disease. Nat. Rev. Mol. Cell Biol. 8, 275-283 (2007)

3. Joerger, A.C., Fersht, A.R.: Structural biology of the tumor suppressor p53. Annu. Rev. Biochem. 77, 557-582 (2008)

4. Yee, K.S., Vousden, K.H.: Complicating the complexity of p53. Carcinogenesis 26, 1317-1322 (2005)

5. Gomes, N.P., Espinosa, J.M.: Differential regulation of p53 target genes: It's (Core Promoter) elementary. Genes Dev. 24, 111-114 (2010)

6. Soussi, T., Defromentel, C.C., May, P.: Structural aspects of the P53 protein in relation to gene evolution. Oncogene 5, 945-952 (1990)

7. Cho, Y.J., Gorina, S., Jeffrey, P.D., Pavletich, N.P.: Crystal-structure of a P53 tumor-suppressor DNA complex-understanding tumorigenic mutations. Science 265, 346-355 (1994)

8. Delphin, C., Cahen, P., Lawrence, J.J., Baudier, J.: Characterization of baculovirus recombinant wild-type P53 - dimerization of P53 is required for high-affinity DNA-binding and cysteine oxidation inhibits P53 DNA-binding. Eur. J. Biochem. 223, 683-692 (1994)

9. Sun, X.Z., Vinci, C., Makmura, L., Han, S.B., Tran, D., Nguyen, J., Hamann, M., Grazziani, S., Sheppard, S., Gutova, M., Zhou, F.M., Thomas, J., Momand, J.: Formation of disulfide bond in p53 Correlates with inhibition of DNA binding and tetramerization. Antioxid. Redox Signal. 5, 655-665 (2003)

10. Parks, D., Bolinger, R., Mann, K.: Redox state regulates binding of p53 to sequence-specific DNA, but not to nonspecific or mismatched DNA. Nucleic Acids Res. 25, 1289-1295 (1997)

11. Buzek, J., Latonen, L., Kurki, S., Peltonen, K., Laiho, M.: Redox State of tumor suppressor p53 regulates its sequence-specific DNA binding in DNA-damaged cells by cysteine 277. Nucleic Acids Res. 30, 2340-2348 (2002)

12. Rainwater, R., Parks, D., Anderson, M.E., Tegtmeyer, P., Mann, K.: Role of cysteine residues in regulation of P53 function. Mol. Cell. Biol. 15, 3892-3903 (1995)

13. Augustyn, K.E., Merino, E.J., Barton, J.K.: A role for DNA-mediated charge transport in regulating p53: oxidation of the DNA-bound protein from a distance. Proc. Natl. Acad. Sci. U.S.A. 104, 18907-18912 (2007) 
14. Wu, H.H., Momand, J.: Pyrrolidine dithiocarbamate prevents p53 activation and promotes 53 Cysteine residue oxidation. J. Biol. Chem. 273, 18898-18905 (1998)

15. Seo, Y.R., Kelley, M.R., Smith, M.L.: Selenomethionine regulation of p53 by a ref1-dependent redox mechanism. Proc. Natl Acad. Sci. USA 99, 14548-14553 (2002)

16. Held, J.M., Danielson, S.R., Behring, J.B., Atsriku, C., Britton, D.J., Puckett, R.L., Schilling, B., Campisi, J., Benz, C.C., Gibson, B.W.: Targeted quantitation of site-specific cysteine oxidation in endogenous proteins using a differential alkylation and multiple reaction monitoring mass spectrometry approach. Mol Cell Proteom 9, 1400$1410(2010)$

17. Hainaut, P., Mann, K.: Zinc binding and redox control of p53 structure and function. Antioxid. Redox Signal. 3, 611-623 (2001)

18. Stoner, C.S., Pearson, G.D., Koc, A., Merwin, J.R., Lopez, N.I., Merrill, G.F.: Effect of thioredoxin deletion and p53 cysteine replacement on human p53 activity in wild-type and thioredoxin reductase null yeast. Biochemistry 48, 9156-9169 (2009)

19. Liu, B., Chen, Y.M., Clair, D.K.S.: ROS and p53: a versatile partnership. Free Radical Biol. Med. 44, 1529-1535 (2008)

20. Meplan, C., Richard, M.J., Hainaut, P.: Redox signaling and transition metals in the control of the p53 pathway. Biochem. Pharmacol. 59, 25 33 (2000)

21. Bykov, V.J.N., Lambert, J.M.R., Hainaut, P., Wiman, K.G.: Mutant p53 rescue and modulation of p53 redox state. Cell Cycle 8, 2509-2517 (2009)

22. Lambert, J.M.R., Gorzov, P., Veprintsev, D.B., Soderqvist, M., Segerback, D., Bergman, J., Fersht, A.R., Hainaut, P., Wiman, K.G., Bykov, V.J.N.: PRIMA-1 reactivates mutant p53 by covalent binding to the core domain. Cancer Cell 15, 376-388 (2009)

23. Marshall, A.G., Hendrickson, C.L., Jackson, G.S.: Fourier transform ion cyclotron resonance mass spectrometry: A primer. Mass Spectrom. Rev. 17, 1-35 (1998)

24. Wells, J.M., McLuckey, S.A.: Collision-induced dissociation (CID) of peptides and proteins. Biol. Mass Spectrom. 402, 148-185 (2005)

25. Zubarev, R.A., Horn, D.M., Fridriksson, E.K., Kelleher, N.L., Kruger, N.A., Lewis, M.A., Carpenter, B.K., McLafferty, F.W.: Electron capture dissociation for structural characterization of multiply charged protein cations. Anal. Chem. 72, 563-573 (2000)

26. Zubarev, R.A., Kelleher, N.L., McLafferty, F.W.: Electron capture dissociation of multiply charged protein cations. A nonergodic process. J. Am. Chem. Soc. 120, 3265-3266 (1998)

27. Kelleher, N.L.: Top-Down Proteomics. Anal Chem 76, 196A-203A (2004)

28. Siuti, N., Kelleher, N.L.: Decoding protein modifications using topdown mass spectrometry. Nat Methods 4, 817-821 (2007)

29. Bogdanov, B., Smith, R.D.: Proteomics by FTICR Mass spectrometry: Top down and bottom up. Mass Spectrom. Rev. 24, 168-200 (2005)

30. Meng, F.Y., Forbes, A.J., Miller, L.M., Kelleher, N.L.: Detection and localization of protein modifications by high resolution tandem mass spectrometry. Mass Spectrom. Rev. 24, 126-134 (2005)

31. Bhanoori, M., Yellaturu, C.R., Ghosh, S.K., Hassid, A., Jennings, L.K., Rao, G.N.: Thiol alkylation inhibits the mitogenic effects of plateletderived growth factor and renders it proapoptotic via activation of STATs and p53 and induction of expression of caspase1 and p21(waf1/ cip1). Oncogene 22, 117-130 (2003)

32. Bykov, V.J.N., Issaeva, N., Zache, N., Shilov, A., Hultcrantz, M., Bergman, J., Selivanova, G., Wiman, K.G.: Reactivation of mutant p53 and induction of apoptosis in human tumor cells by maleimide analogs. J. Biol. Chem. 280, 30384-30391 (2005)

33. Patel, S., Bui, T.T.T., Drake, A.F., Fraternali, F., Nikolova, P.V.: The p73 DNA binding domain displays enhanced stability relative to Tts homologue, the tumor suppressor p53, and exhibits cooperative DNA binding. Biochemistry 47, 3235-3244 (2008)
34. Patel, S., George, R., Autore, F., Fraternali, F., Ladbury, J.E., Nikolova, P.V.: Molecular interactions of ASPP1 and ASPP2 with the $\mathrm{p} 53$ protein family and the apoptotic promoters PUMA and Bax. Nucleic Acids Res. 36, 5139-5151 (2008)

35. Bullock, A.N., Henckel, J., DeDecker, B.S., Johnson, C.M., Nikolova, P.V., Proctor, M.R., Lane, D.P., Fersht, A.R.: Thermodynamic stability of wild-type and mutant p53 core domain. Proc. Natl. Acad. Sci. U.S.A. 94, 14338-14342 (1997)

36. Pesavento, J.J., Mizzen, C.A., Kelleher, N.L.: Quantitative analysis of modified proteins and their positional isomers by tandem mass spectrometry: human histone H4. Anal. Chem. 78, 4271-4280 (2006)

37. LeDuc, R.D., Taylor, G.K., Kim, Y.B., Januszyk, T.E., Bynum, L.H., Sola, J.V., Garavelli, J.S., Kelleher, N.L.: ProSight PTM: an integrated environment for protein identification and characterization by top-down mass spectrometry. Nucleic Acids Res. 32, W340-W345 (2004)

38. Meplan, C., Richard, M.J., Hainaut, P.: Metalloregulation of the tumor suppressor protein p53: zinc mediates the renaturation of p53 after exposure to metal chelators in vitro and in intact cells. Oncogene 19, 5227-5236 (2000)

39. Butler, J.S., Loh, S.N.: Structure, function, and aggregation of the zincfree form of the p53 DNA binding domain. Biochemistry 42, 2396$2403(2003)$

40. Khoo, K.H., Mayer, S., Fersht, A.R.: Effects of stability on the biological function of p53. J. Biol. Chem. 284, 30974-30980 (2009)

41. Friedler, A., Veprintsev, D.B., Hansson, L.O., Fersht, A.R.: Kinetic instability of p 53 core domain mutants: Implications for rescue by small molecules. J. Biol. Chem. 278, 24108-24112 (2003)

42. Boyne, M.T., Garcia, B.A., Li, M.X., Zamdborg, L., Wenger, C.D., Babai, S., Kelleher, N.L.: Tandem mass spectrometry with ultrahigh mass accuracy clarifies peptide identification by database retrieval. $J$. Proteome Res. 8, 374-379 (2009)

43. Wang, Y., Rosengarth, A., Luecke, H.: Structure of the human p53 core domain in the absence of DNA. Acta Crystallogr. D Biol. Crystallogr. 63, 276-281 (2007)

44. Kaar, J. L.; Basse, N.; Joerger, A. C.; Stephens, E.; Rutherford, T. J.; Fersht, A. R. Stabilization of Mutant p53 Via Alkylation of Cysteines and Effects on DNA Binding. Protein Sci. 2010, Epub ahead of print.

45. Joerger, A.C., Allen, M.D., Fersht, A.R.: Crystal structure of a superstable mutant of human p53 core domain. Insights into the mechanism of rescuing oncogenic mutations. J. Biol. Chem. 279, 1291-1296 (2004)

46. Velu, C.S., Niture, S.K., Doneanu, C.E., Pattabiraman, N., Srivenugopal, K.S.: Human p53 is inhibited by glutathionylation of cysteines present in the proximal DNA-binding domain during oxidative stress. Biochemistry 46, 7765-7780 (2007)

47. Haupt, S., Berger, M., Goldberg, Z., Haupt, Y.: Apoptosis-the p53 network. J. Cell Sci. 116, 4077-4085 (2003)

48. Kim, D.H., Kim, E.H., Na, H.K., Sun, Y., Surh, Y.J.: 15-Deoxy-delta $(12,14)$-prostaglandin $J(2)$ stabilizes, but functionally inactivates $\mathrm{p} 53$ by binding to the cysteine 277 residue. Oncogene 29, 2560-2576 (2010)

49. Craig, A.L., Chrystal, J.A., Fraser, J.A., Sphyris, N., Lin, Y., Harrison, B.J., Scott, M.T., Dornreiter, I., Hupp, T.R.: The MDM2 Ubiquitination signal in the DNA-binding domain of p53 forms a docking site for calcium calmodulin kinase superfamily members. Mol. Cell. Biol. 27, 3542-3555 (2007)

50. Glineur, C., Davioud-Charvet, E., Vandenbunder, B.: The conserved redox-sensitive cysteine residue of the DNA-binding region in the c-Rel protein is involved in the regulation of the phosphorylation of the protein. Biochem. J. 352, 583-591 (2000)

51. D'Autreaux, B., Toledano, M.B.: ROS as signaling molecules: mechanisms that generate specificity in ROS homeostasis. Nature Rev. Mol. Cell Biol. 8, 813-824 (2007) 